\title{
Non-splitting Tridiagonalization of Complex Symmetric Matrices
}

\author{
W.N. Gansterer ${ }^{1}$, A.R. Gruber ${ }^{2}$, and C. Pacher ${ }^{3}$ \\ 1 University of Vienna, Research Lab Computational Technologies and Applications \\ wilfried.gansterer@univie.ac.at \\ 2 University of Vienna, Institute for Theoretical Chemistry \\ agruber@tbi.univie.ac.at \\ 3 Austrian Research Centers GmbH-ARC, Department of Safety \& Security \\ christoph.pacher@arcs.ac.at
}

\begin{abstract}
A non-splitting method for tridiagonalizing complex symmetric (non-Hermitian) matrices is developed and analyzed. The main objective is to exploit the purely structural symmetry in terms of runtime performance. Based on the analytical derivation of the method, Fortran implementations of a blocked variant are developed and extensively evaluated experimentally. In particular, it is illustrated that a straightforward implementation based on the analytical derivation exhibits deficiencies in terms of numerical properties. Nevertheless, it is also shown that the blocked non-splitting method shows very promising results in terms of runtime performance. On average, a speed-up of more than three is achieved over competing methods. Although more work is needed to improve the numerical properties of the non-splitting tridiagonalization method, the runtime performance achieved with this non-unitary tridiagonalization process is very encouraging and indicates important research directions for this class of eigenproblems.
\end{abstract}

Keywords: Tridiagonalization, complex symmetric eigenvalue problems, complex symmetric reflector.

\section{Introduction}

We discuss an algorithm for tridiagonalizing a complex symmetric (non Hermitian) matrix $C \in \mathbb{C}^{n \times n}$. This task is a central component in reduction methods for solving the complex symmetric eigenvalue problem (EVP)

$$
C x=\lambda x \quad \text { with } \quad C \in \mathbb{C}^{n \times n}, C=C^{\top} .
$$

Problems of this type are a special case of general non Hermitian complex eigenproblems. Although they do not occur as frequently in practice as real symmetric or complex Hermitian problems, there are many important applications where they arise [1. An example is the numerical solution of Maxwell's equations with complex material coefficients (accounting for losses) and/or certain absorbing boundary conditions used in the simulation of optoelectronic devices 2 .

G. Allen et al. (Eds.): ICCS 2009, Part I, LNCS 5544, pp. 481-490, 2009.

(C) Springer-Verlag Berlin Heidelberg 2009 
Especially for large $n$, it is pivotal to exploit the (non Hermitian) symmetry present in problem (11) in order to be able to efficiently solve such problems.

Hardly any high quality software is available for complex symmetric eigenproblems. Only QMRPACK [3] contains an implementation of a complex symmetric Lanczos algorithm. General purpose state-of-the-art software libraries for dense numerical linear algebra computations, such as the BLAS 4] and LAPACK 5] for sequential computations, or the parallel packages SCALAPACK 6] and PLAPACK [7, contain very few computational routines which are capable of specifically exploiting complex symmetry. No such routine for the complex symmetric eigenvalue problem is currently available in these state-of-the-art software packages. Consequently, the currently most common strategy for solving problems (11) is to ignore their special properties and to solve them with the technology available for general non Hermitian problems (for example, using the routine LAPACK/zgeev): The complex symmetric matrix $C$ is first reduced to Hessenberg form using unitary transformations, from which eigenvalues and eigenvectors are computed by applying standard methods for unsymmetric matrices. This strategy has obvious disadvantages in terms of computational effort and in terms of storage requirements.

The main objective of this paper is to investigate a non-splitting approach for tridiagonalizing $C$ and to compare it on the one hand to standard methods for general non Hermitian eigenproblems, and on the other hand to the splitting tridiagonalization method for complex symmetric matrices discussed earlier [2].

Related Work. Various projection methods for solving complex symmetric EVPs have been proposed, for example, based on modifications of the nonHermitian Lanczos method [389], on subspace iteration [10], or on variants of the Jacobi-Davidson method [11.

For dense matrices and/or if large parts of the spectrum are to be computed, transformation methods (based on tridiagonalization) can be more efficient [12. For these methods, the tridiagonalization step tends to be a dominating part in terms of arithmetic complexity and usually also computation time. Earlier attempts were based on modifying the conventional Householder-based tridiagonalization for real symmetric or complex Hermitian matrices such that symmetry is preserved for complex symmetric problems [13]. In [13], the Householder vector is normalized using a quasi-inner product, which has several implications: The normalization factor can become a negative or a complex number, and the resulting transformation matrices are not unitary. The idea of tridiagonalizing real and imaginary part of $C$ separately, connected by complex orthogonal transformations (called splitting method in the following) for tridiagonalizing a complex symmetric matrix has been investigated in [142].

For computing eigenvalues and eigenvectors of the resulting tridiagonal complex symmetric problem, modifications of the $Q R$ algorithm [15]16] and the routines cmtql1 and inverm [17] have been used.

In this paper, we investigate non-splitting methods as an alternative approach for tridiagonalizing a complex symmetric matrix $C$. The basic concept is very similar to the one mentioned in [13. However, in 13 neither a discussion of 
numerical properties (observed deficiencies in accuracy, special techniques for overcoming them, etc.) nor a quantitative performance evaluation of this approach is given. We provide a comparison of this approach to the splitting method in terms of numerical properties and runtime performance.

Synopsis. In Section 2 we review general properties of complex symmetric matrices and we summarize the splitting method introduced earlier. In Section3, we derive a non-splitting tridiagonalization method for complex symmetric matrices and relate it to generalizations of unitary Householder reflectors. In Section 4 an experimental evaluation of this non-splitting method and a comparison to other approaches is summarized, and Section 5 contains conclusions and future work.

\section{Background}

Mathematically speaking, structural symmetry is not a very distinctive feature of complex matrices, since every matrix $A \in \mathbb{C}^{n \times n}$ is similar to a complex symmetric matrix [1. In contrast to a real symmetric matrix, a complex symmetric matrix $A$ is not necessarily normal and not necessarily diagonalizable [1]. Nevertheless, the purely algebraic symmetry is of great interest for the development of space- and time-efficient algorithms. Obviously, half of the information in a complex symmetric matrix is redundant, and efficient algorithms should be able to take advantage of this fact in terms of memory requirements as well as in terms of computational effort.

\subsection{Complex Orthogonal Transformations}

As a basic guideline, there are two conditions for any transformation used in the tridiagonalization process of $C$ : $(i)$ It has to be a similarity transformation in order to preserve the spectrum of $C$, and ( $i i)$ it has to be symmetry-preserving in order to exploit the structural symmetry. Consequently, given a transformation matrix $G \in \mathbb{C}^{n \times n}$, each transformation needs to be of the form $G C G^{-1}$ in order to satisfy the first condition and of the form $G C G^{\top}$ in order to satisfay the second condition. In summary, the basic transformation matrices used need to satisfy

$$
G G^{\top}=I,
$$

which defines a complex orthogonal transformation [1] (COT) $G$. Note that $G$ is not unitary and in general $\|G\|_{2}>1$. Consequently, the application of complex orthogonal transformations $G$ potentially involves compromising numerical stability. In order to bound the numerical errors in transformation processes using complex orthogonal matrices, their norms have to be monitored, and, if possible, kept below some properly chosen threshold.

\subsection{The Splitting Method}

The splitting method, which has been introduced in 14, is based on separating the tridiagonalization of the real part $R$ of $C$ from the tridiagonalization of the 
imaginary part $S$ of $C$ ( $R$ and $S$ are both real symmetric matrices) as much as possible. For example, the part below the subdiagonal of a column of $R$ (say, of length $k$ ) can be eliminated using a real orthogonal transformation matrix $Q_{\mathrm{R}}$. After that, a $k-1$ part of the corresponding column of $S$ can be eliminated without causing any fill-in in $R$ using another real orthogonal matrix $Q_{\mathrm{I}}$. Both of these operations are performed in real arithmetic, and both transformation matrices have norm one. Eventually, a single nonzero element below the subdiagonal in $S$ remains to be eliminated. This operation has to be performed in complex arithmetic, using a $2 \times 2$ complex orthogonal transformation matrix, whose norm cannot be bounded a priori.

In [2], we have investigated numerical properties and runtime performance of the splitting method for tridiagonalizing $C$, and we have pointed out some algorithmic variants which have not been mentioned in 14. Our experimental results showed that the splitting method can achieve good numerical accuracy, but the runtime performance achieved was often not better than the one achieved with the routine zgeev from LAPACK [5].

\section{Non-splitting Tridiagonalization Methods}

Non-splitting methods for tridiagonalizing $C$ are characterized by the fact that, in contrast to the splitting method, they do not split up $C$ into its real and imaginary parts but operate in complex arithmetic on the complex symmetric matrix as a whole. The method investigated in this paper can be derived from an old method proposed by La Budde [18] for tridiagonalizing any real unsymmetric matrix. In the following, we first briefly review La Budde's method and then show how it can be modified to tridiagonalize a complex symmetric matrix.

\subsection{La Budde's Method}

In order to eliminate a column vector $c \in \mathbb{R}^{n-j}$ below the subdiagonal and a row vector $b \in \mathbb{R}^{n-j}$ right of the superdiagonal in an unsymmetric real $n \times n$ matrix, La Budde [18] determined elimination vectors $x, y \in \mathbb{R}^{n-j}$, such that the matrices

$$
\begin{array}{ll}
M_{\alpha}:=I_{n-j}+\alpha x y^{\top}, & \alpha \neq 0, \\
M_{\beta}:=I_{n-j}+\beta x y^{\top}, & \beta \neq 0,
\end{array}
$$

constitute a similarity transformation $\left(M_{\alpha} M_{\beta}=I_{n-j}\right)$ and eliminate all entries except the first one in the vectors $c$ and $b$ :

$$
\begin{aligned}
M_{\alpha} c & =\tau_{1} e_{1}, \\
b^{T} M_{\beta} & =\tau_{2} e_{1}^{T},
\end{aligned}
$$

where $e_{1}=(1,0, \ldots, 0)^{T} \in \mathbb{R}^{n-j}$. Carrying out this process over columns $j=$ $1,2, \ldots, n-2$, the given matrix can be transformed to tridiagonal form. 
It turns out that La Budde's method breaks down when at some point in the process $s:=b^{\top} c=0$. La Budde suggested in [18] to avoid this breakdown by a proper choice of the parameters $\alpha$ and $\beta$ in (3) and (44). However, it was pointed out in [19] that there are at least two categories of matrices where it is not possible to choose $\alpha$ and $\beta$ such that recovery from $s=0$ is possible. Remedies were proposed for these cases. Parlett [20] has pointed out that La Budde's procedure applied to Hessenberg matrices is identical to well-known transformation methods. He also showed that for Hessenberg matrices $s$ is invariant for all permissible choices of $\alpha$ and $\beta$. Therefore, Hessenberg matrices form another class of matrices where recovery from breakdown is not possible by manipulating $\alpha$ and $\beta$.

\subsection{Adaptation for Complex Symmetric Matrices}

In the complex symmetric case $b, c, x, y$ are complex vectors and $c=b, \alpha=\beta$, $x=y$. Therefore, $M_{\alpha}=M_{\beta}$ and $M_{\alpha}=M_{\alpha}^{T}$. This yields the following equations:

$$
\begin{aligned}
M_{\alpha} & =I_{n-j}+\alpha x x^{T}, \quad \alpha \neq 0, \\
M_{\alpha}^{2} & =I_{n-j}, \\
M_{\alpha} c & =\tau e_{1} .
\end{aligned}
$$

From (5) and (6) and from the fact that in the nontrivial case $M_{\alpha} \neq I_{n-j}$ we can conclude $\alpha=-2 / x^{T} x$ and thus

$$
M_{\alpha}=I_{n-j}-\frac{2}{x^{T} x} x x^{T} .
$$

Note that in the symmetric case $M_{\alpha}$ does in fact not depend on $\alpha$ any more and thus we drop the index $\alpha$ in the following.

Now, the vector $x$ has to be determined so that (7) is satisfied. From (7) we obtain $\tau^{2}=\tau e_{1}^{T} e_{1} \tau=c^{T} M^{T} M c=c^{T} c$, where the last equation follows from $M^{T} M=M M=I$. Consequently

$$
\tau= \pm \sqrt{c^{T} c}
$$

and the resulting vector

$$
\tau e_{1}= \pm \sqrt{c^{T} c} e_{1}
$$

Thus, we have

$$
M c= \pm \sqrt{c^{T} c} e_{1} \Leftrightarrow \quad c-\frac{2}{x^{T} x}\left(x^{T} c\right) x= \pm \sqrt{c^{T} c} e_{1}
$$

which we must solve for $x$. Note that $x$ can be scaled by any (complex) number $a$ without changing $M$. If we scale $x$ such that $\frac{2}{x^{T} x}\left(x^{T} c\right)=1$ holds, then the following solutions are easily found:

$$
x=c \mp \sqrt{c^{T} c} e_{1} .
$$


Consequently, we obtain the following equations for the components of the elimination vector $x$ :

$$
\begin{aligned}
& x_{1}=c_{1} \mp \sqrt{c^{T} c}=c_{1}-\tau, \\
& x_{k}=c_{k}, \quad k=2, \ldots, n .
\end{aligned}
$$

Note that this is analogous to the definition of the real Householder reflector. This shows on the one hand that in the real symmetric case La Budde's method is identical to real Householder reflectors, and on the other hand that in the complex symmetric case it leads to a direct generalization of Householder reflectors in complex arithmetic.

\subsection{Numerical Properties}

Since for a vector $x \in \mathbb{C}^{n-j}, x^{\top} x=0$ is possible even if $x \neq 0$, (8) indicates that $\|M\| \geq 1$ and that breakdown and numerical problems are possible. For the splitting method, various recovery transformations for controlling and improving numerical accuracy have already been investigated [14]. Our derivation above illustrates that the need for monitoring and improving numerical accuracy also arises in the context of non-splitting methods. The investigation and development of suitable recovery transformations in this context is topic of ongoing work. In the following section, we evaluate a straightforward implementation of the basic non-splitting method based on (9) and (10).

\section{Experimental Evaluation}

For our experiments, we implemented the following routines in Fortran: zsytd2 performs an unblocked non-splitting tridiagonalization of a complex-symmetric matrix $C$, zsytrd performs a blocked non-splitting tridiagonalization of complexsymmetric $C$, zunm2r2 performs an unblocked backtransformation of the eigenvectors of the tridiagonal matrix $T$ to those of $C$, and zunmtr2 performs a blocked backtransformation of the eigenvectors of the tridiagonal matrix $T$ to those of $C$. The routines compev [17] and inverm [17] were used for computing eigenvalues and corresponding eigenvectors of the complex symmetric tridiagonal matrix. For comparison, the routine LAPACK/zgeev for general non Hermitian eigenvalue problems was used.

The codes were run on a Sun Fire v40z with 4 dual-core Opteron 875 CPUs $(2.2 \mathrm{GHz})$ and $24 \mathrm{~GB}$ main memory. Suse Linux Enterprise Server 10, the GNU Fortran 95 compiler, LAPACK version 3.1.1 and Goto BlAS 1.20 were used. The test matrices were created randomly.

\subsection{Numerical Accuracy}

Denoting with $\left(\lambda_{i}, x_{i}\right)$ the eigenpairs computed by LAPACK/zgeev, and with $\left(\tilde{\lambda}_{i}, \tilde{x}_{i}\right)$ the eigenpairs computed by non-splitting tridiagonalization followed by 


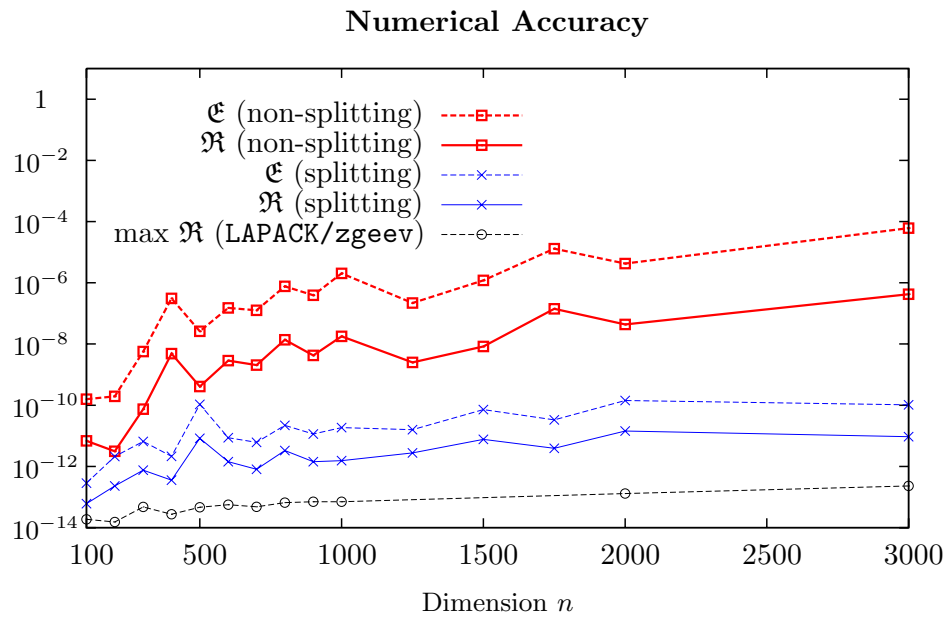

Fig. 1. Residuals and eigenvalue errors for different complex symmetric eigensolvers

compev and inverm, an average eigenvalue error $\mathfrak{E}$ and an average residual error $\mathfrak{R}$ have been computed according to

$$
\mathfrak{E}:=\operatorname{average}_{i} \frac{\left|\tilde{\lambda}_{i}-\lambda_{i}\right|}{\left|\lambda_{i}\right|}, \quad \mathfrak{R}=\operatorname{average}_{i} \frac{\left\|\left(A-\tilde{\lambda}_{i} I_{n}\right) \tilde{x}_{i}\right\|_{2}}{\|A\|_{2}}, \quad i \in\{1, \ldots, n\} .
$$

Fig. 1]illustrates the experimental results. It has already been shown in [2] that the error introduced by the splitting tridiagonalization (without the solver for the tridiagonal problem) is only about two orders of magnitudes higher than the one of LAPACK/zgeev. The first straightforward implementation of the non-splitting method used here looses some more orders of magnitude in accuracy compared to the splitting method. (Note that for LAPACK/zgeev the maximum residuals are shown, not their averages.) This indicates the need for improvements of the numerical properties of the non-splitting tridiagonalization approach, as already mentioned in Section 3.3. Corresponding efforts are work in progress.

\subsection{Runtime Performance}

In 2 we have already illustrated that with the GOTO BLAS, LAPACK/zgeev tends to outperform the splitting method in terms of runtime performance. Thus, in Fig. 2 we compare normalized runtimes of blocked non-splitting tridiagonalization followed by compev, inverm and blocked backtransformation (the sum of these runtimes is denoted by nonsplit) to normalized runtimes of LAPACK/zgeev. Fig. 2]illustrates the big potential of non-splitting tridiagonalization: Its runtime performance is significantly better than the one of LAPACK/zgeev and consequently also than the one of the splitting method. Despite a similar asymptotic behavior, the non-splitting approach achieves on average a speed-up of a little more than three over LAPACK/zgeev. 


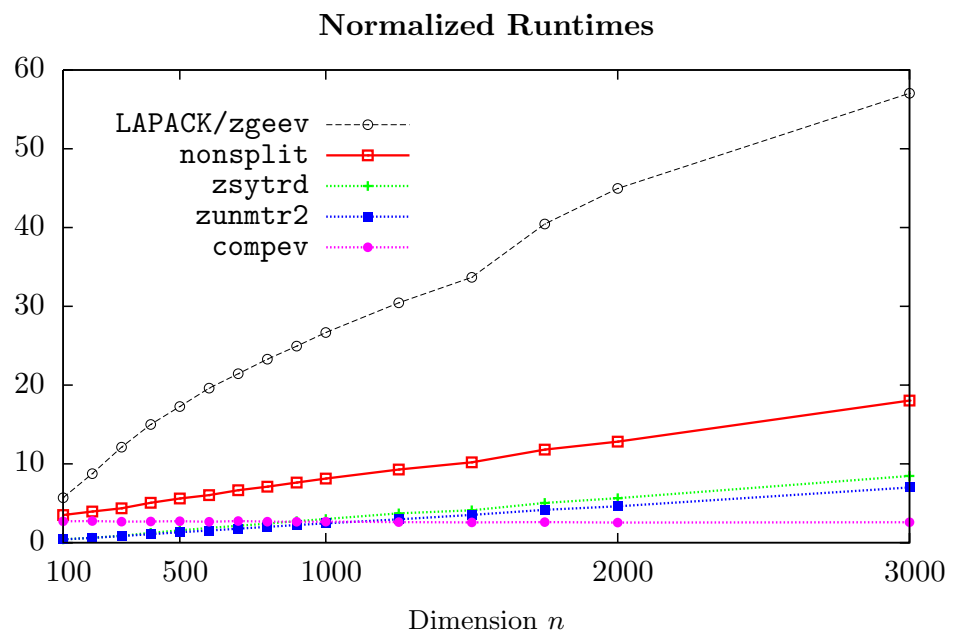

Fig. 2. Normalized runtimes $\left(T(n) / n^{2}\right)$ of complex symmetric eigensolver (nonsplit) based on non-splitting tridiagonalization, of its components, and of LAPACK/zgeev

This underlines the importance of efforts in improving the numerical properties of the non-splitting approach. If they can be improved by some properly chosen recovery transformations in cases where the naive implementation used here suffers from numerical inaccuracies, then a central building block can be established for a very competitive method for solving dense complex symmetric eigenproblems (1).

\section{Conclusions and Future Work}

A non-splitting tridiagonalization process for complex symmetric matrices which can be derived from a method for tridiagonalizing unsymmetric matrices originally suggested in [18, has been investigated. It has been shown that this approach leads to a symmetry preserving generalization of complex Householder reflectors. The non-splitting tridiagonalization process and a complex symmetric eigensolver based on it have been analyzed in terms of numerical properties and runtime performance.

Compared to the standard LAPACK routine for general non Hermitian eigenproblems, LAPACK/zgeev, and to the splitting method proposed and analyzed earlier 142, the naive straightforward implementation of the non-splitting approach exhibits a loss of numerical accuracy (measured in terms of eigenvalue error and in terms of residual error). Potential sources for this loss of accuracy are clear, and work on improving the numerical properties of the non-splitting method is in progress. In terms of runtime performance, a blocked non-splitting approach shows very promising results. In the experiments summarized in this paper, on average a speed-up of more than three could be achieved over competing methods. If progress can be made in the numerical aspects, then non-splitting 
methods have the potential to become a standard approach for tridiagonalizing complex symmetric matrices.

The work summarized here motivates various further research directions. As mentioned before, the improvement of the numerical properties of non-splitting tridiagonalization is of utmost importance. Once this aspect is sucessfully addressed, it should be possible to develop parallelization strategies analogously to Householder-based tridiagonalization methods for real symmetric or complex Hermitian matrices.

\section{References}

1. Horn, R.A., Johnson, C.R.: Matrix Analysis. Cambridge University Press, Cambridge (1985)

2. Gansterer, W.N., Schabauer, H., Pacher, C., Finger, N.: Tridiagonalizing complex symmetric matrices in waveguide simulations. In: Bubak, M., van Albada, G.D., Dongarra, J., Sloot, P.M.A. (eds.) ICCS 2008, Part I. LNCS, vol. 5101, pp. 945-954. Springer, Heidelberg (2008)

3. Freund, R.W., Nachtigal, N.M.: QMRPACK: a package of QMR algorithms. ACM Trans. Math. Softw. 22(1), 46-77 (1996)

4. Dongarra, J.J., Du Croz, J., Duff, I.S., Hammarling, S.: A set of level 3 basic linear algebra subprograms. ACM Trans. Math. 16, 1-17, 18-28 (1990)

5. Anderson, E., Bai, Z., Bischof, C.H., Blackford, S., Demmel, J.W., Dongarra, J.J., Du Croz, J., Greenbaum, A., Hammarling, S., McKenney, A., Sorensen, D.C.: Lapack Users Guide, 3rd edn. SIAM Press, Philadelphia (1999)

6. Blackford, L.S., Choi, J., Cleary, A., D’Azevedo, E., Demmel, J.W., Dhillon, I., Dongarra, J.J., Hammarling, S., Henry, G., Petitet, A., Stanley, K., Walker, D., Whaley, R.C.: ScaLapack Users' Guide. SIAM Press, Philadelphia (1997)

7. van de Geijn, R.: Using PLapack: Parallel Linear Algebra Package. The MIT Press, Cambridge (1997)

8. Freund, R.W., Gutknecht, M.H., Nachtigal, N.M.: An implementation of the lookahead Lanczos algorithm for non-hermitian matrices. SIAM J. Sci. Comput. 14(1), 137-158 (1993)

9. Cullum, J.K., Willoughby, R.A.: A practical procedure for computing eigenvalues of large sparse nonsymmetric matrices. In: Cullum, J.K., Willoughby, R.A. (eds.) Proceedings of the IBM Europe Institute Workshop on Large Scale Eigenvalue Problems, pp. 193-223. North-Holland, Amsterdam (1986)

10. Leung, A.Y.T.: Subspace iteration for complex symmetric eigenproblems. J. Sound Vibration 184(4), 627-637 (1995)

11. Arbenz, P., Hochstenbach, M.E.: A Jacobi-Davidson method for solving complex symmetric eigenvalue problems. SIAM J. Sci. Comput. 25(5), 1655-1673 (2004)

12. Bai, Z., Demmel, J., Dongarra, J.J., Ruhe, A., van der Vorst, H. (eds.): Templates for the Solution of Algebraic Eigenvalue Problems: A Practical Guide. SIAM Press, Philadelphia (2000)

13. Ohnami, K., Mikami, Y.: Resonance scattering in a two-dimensional non-integrable system. J. Phys. A 25, 4903-4912 (1992)

14. Bar-On, I., Ryaboy, V.: Fast diagonalization of large and dense complex symmetric matrices, with applications to quantum reaction dynamics. SIAM J. Sci. Comput. 18, 1412-1435 (1997) 
15. Cullum, J.K., Willoughby, R.A.: A $Q L$ procedure for computing the eigenvalues of complex symmetric tridiagonal matrices. SIAM J. Matrix Anal. Appl. 17, 83-109 (1996)

16. Luk, F., Qiao, S.: Using complex-orthogonal transformations to diagonalize a complex symmetric matrix. In: Luk, F.T. (ed.) Advanced Signal Processing: Algorithms, Architectures, and Implementations VII, Proc. SPIE, vol. (3162), pp. 418 425 (1997)

17. Cullum, J.K., Willoughby, R.A.: Lanczos Algorithms for Large Symmetric Eigenvalue Computations, vol. 1: Theory, vol. 2: Programs. Birkhäuser, Boston (1985)

18. La Budde, C.D.: The reduction of an arbitrary real square matrix to tridiagonal form using similarity transformations. Math. Comp. 17, 433-437 (1963)

19. Wang, H.H., Gregory, R.T.: On the reduction of an arbitrary real square matrix to tridiagonal form. Math. Comp. 18, 501-505 (1964)

20. Parlett, B.N.: A note on La Budde's algorithm. Math. Comp. 18, 505-506 (1964) 